\title{
HÁBITOS DE CONSUMO DE CARNE BOVINA EN TEMUCO, IX REGIÓN DE CHILE ${ }^{1}$
}

\author{
BEEF CONSUMPTION HABITS IN TEMUCO, IX REGION OF CHILE
}

\author{
Berta Schnettler Morales² ${ }^{2}{\text { Oscar Manquilef Bäschler }{ }^{2} \text {; Horacio Miranda Vargas }}^{2}$
}

\begin{abstract}
RESUMEN
Considerando la importancia del gasto en carne dentro del presupuesto familiar de los hogares chilenos, se determinaron los hábitos, preferencias de consumo y conocimiento del uso de la carne bovina de compradores de supermercados en la ciudad de Temuco, IX Región de Chile, y la existencia de diferencias entre distintos segmentos de población. Se realizó una encuesta en once supermercados a una muestra de 260 personas, estratificada por género, grupo socioeconómico y edad. La carne bovina fue preferida por sobre el pollo y cerdo. La persona que decide y compra la carne bovina correspondió mayoritariamente a mujeres. Predominó la compra de carne bovina en cortes, una frecuencia de consumo tres veces por semana y adquisiciones entre 1 y 2 $\mathrm{kg}$ en cada evento de compra. Aproximadamente 50\% presentó un bajo conocimiento de los usos de los cortes de la carne. Se detectaron diferencias en hábitos, preferencias y grado de conocimiento de los usos culinarios de la carne bovina según el género y estrato socioeconómico del consumidor.
\end{abstract}

Palabras clave: Carne bovina, demanda, segmentación.

\begin{abstract}
Considering the high proportion spend of beef in the budget of Chilean households, a survey carried out that looked at the habits, consumption preferences and knowledge about the use of beef among supermarket clients in Temuco, IX Region of Chile. The existence of differences between various segments of the population was also looked at. The survey was carried out in eleven supermarkets, with a sample of 260 individuals, stratified by sex, socio-economic stratum and age. Beef was preferred to chicken and pork. The individuals who made the decision and purchased the beef were generally women. It was most common for beef to be purchased in cuts, with a frequency of three times per week, and in quantities of between 1 and $2 \mathrm{~kg}$ at each purchase. Approximately 50\% displayed a low level of knowledge with regard to the uses of the various cuts. Differences in habits, preferences and degree of knowledge of the culinary uses of beef were detected according to the sex and socio-economic stratum of the consumer.
\end{abstract}

Key word: Beef, demand, segmentation.

\section{INTRODUCCIÓN}

La carne es un producto de alto precio y ocupa una parte importante del presupuesto destinado a la compra de alimentos en los hogares (West et al., 2001). En Chile $27 \%$ del presupuesto de los hogares se destina a la compra de alimentos, dentro de estos $18 \%$ corresponde a carne (INE, 2001). El consumo de carne en Chile en 2004 fue 76,9 kilos por habitante, con una tasa de crecimiento de $5 \%$ anual en el período 1990-2004. El consumo per cápita de carne bovina, que en 1990 era el más importante entre las carnes, se ubica hoy en segundo lugar después de la carne de ave, con $25,1 \mathrm{~kg}$ y una tasa de crecimiento anual de $2,1 \%$ (ODEPA, 2005). Esta tendencia a la disminución del consumo de carne bovina no sólo ocurre en Chile, sino que en varios países europeos debido a cambios en los gustos y patrones de preferencias de los consumidores (Piggott et al., 1996; Rickertsen, 1996). En EE.UU. los factores más importantes corresponden a un aumento de la preocupación por la salud, cambio en el perfil demográfico, más salidas a comer fuera del

\footnotetext{
1 Realizado en el marco del proyecto DIDUFRO 120601

2 Universidad de La Frontera, Facultad de Ciencias Agropecuarias y Forestales, Casilla 54-D, Temuco, Chile.

E-mail: bschnett@ufro.cl
}

Fecha de Recepción: 16 Marzo 2006

Fecha de Aceptación: 18 Abril 2006 
hogar y cambios en los precios relativos (USDA/ERS, 2002).

A diferencia de otros rubros pecuarios en Chile, como pollos y cerdos, la posesión de ganado bovino está en manos de grandes y pequeños productores. Las cifras del IV Censo Nacional Agropecuario (INE, 1998) señalan la existencia de 160.218 explotaciones dedicadas al rubro bovino con 4.098.432 cabezas; $90 \%$ de las explotaciones posee menos de 100 ha y $44 \%$ de las existencias de ganado, mientras $10 \%$ de las explotaciones con superficies superiores a 100 ha cada una concentra $56 \%$ de la masa ganadera bovina.

El mercado de la carne bovina en Chile ha experimentado numerosos cambios en el último tiempo, con disminuciones en el consumo y la producción (carne en vara: 242.452 t en 1990, 208.259 en 2004) e incremento de las importaciones (2.867 $t$ en 1990; $126.224 t$ en 2004). Las exportaciones de carne bovina adquieren importancia a partir de 2002, registrándose envíos por 8.363 t en 2004 y precios sobre US\$FOB 2,0 $\mathrm{kg}^{-1}$ los tres últimos años (ODEPA, 2005). Además, en el ámbito nacional se ha seguido la tendencia de los países desarrollados, en que los supermercados han ido desplazando a las carnicerías de barrio. Así en el período 1996-97 las ventas de carne bovina a través de supermercados alcanzaron a 57\% (Maino et al., 2000).

Existen numerosos factores asociados al consumo de alimentos, dentro de los cuales destacan las variables demográficas como la edad, género, segmento socioeconómico, educación e ingreso de los consumidores (Burton et al., 1994; Greatorex y Mitchell, 1994; Gilbert y Warren, 1995). Generalmente la demanda y consumo de carne se asocia con el comportamiento del precio y del ingreso de los consumidores (Chalfant y Alston, 1988; Heiman et al., 2001). Los cambios en las características demográficas de los consumidores han conducido a variaciones en el consumo de carnes rojas, puesto que el crecimiento del ingreso y cambios en las características demográficas afectan el número de alimentos demandados por los consumidores (Resurreccion, 2003). Heiman et al. (2001), en un estudio de consumo de carne en Israel, determinaron que las preferencias familiares determinan la cantidad consumida de cada carne.

West et al. (2001) determinaron que la frecuencia de compra de carne bovina y la importancia del precio como atributo de compra son más influidas por los hábitos, edad y sexo de los consumidores, que por el precio del producto y el ingreso y educación del consumidor. En Inglaterra se han detectado diferencias en el consumo de carne dependiendo del género, segmento socioeconómico y diferencias generacionales: las mujeres consumen $45 \%$ menos carne bovina a la semana que los hombres, los segmentos de mayores ingresos tienden a preferir ciertos cortes y el consumo tiende a disminuir con la edad (MINTEL, 1997; MAFF, 1998). Becker et al. (1998) indican que la disminución del consumo de carne con la edad se relaciona con cambios de estilo de vida, gustos y patrones de preferencia asociados al paso de los años.

En base a estos antecedentes el presente estudio tuvo como objetivo determinar hábitos, preferencias de consumo y conocimiento del uso de carne bovina, como asimismo la existencia de diferencias entre distintos segmentos de población en compradores de supermercados de la ciudad de Temuco, IX Región de La Araucanía.

\section{MATERIALES Y MÉTODOS}

El diseño del presente estudio fue observacional, de tipo transversal exploratorio. Se aplicaron encuestas a una muestra compuesta por personas de género femenino o masculino que tuvieran más de 20 años, sin límite superior de edad. El tamaño muestral se calculó en base a la población estimada por el INE para la ciudad de Temuco, correspondiente a 253.868 habitantes para el año 2002. Se utilizó una adaptación de la fórmula estadística presentada por Scheaffer et al. (1996), referida a muestreo probabilístico irrestricto aleatorio. De esta forma, la muestra quedó compuesta por 260 personas, determinando un error de $5 \%$ y una confiabilidad de $95 \%$. La selección de las personas a encuestar se realizó al azar a la entrada de once supermercados de la ciudad de Temuco, ubicados en sectores con diferentes características socioeconómicas, de los cuales el de menor superficie construida presentó $1.600 \mathrm{~m}^{2}$. Previo a la realización de las preguntas de la encuesta, se consultó la edad, género y nivel de ingreso mensual del hogar, esta última característica con el objeto de complementar la información respecto a los estratos socioeconómicos. La encuesta se aplicó de manera uniforme en cuanto a esta variable para acceder de forma similar a cada uno de ellos. Según la edad, los encuestados fueron divididos en once grupos etarios, desde 20 años hasta 70 años y más, abarcando cada grupo cinco edades a excepción del 
último. La muestra así obtenida resultó constituida según género por 83 hombres y 177 mujeres; según el estrato socioeconómico por 56 personas de los estratos $\mathrm{ABC} 1$ (alto y medio alto), 60 personas del estrato C2 (medio-medio), 46 personas del estrato C3 (medio-bajo), 43 personas del estrato D (bajo) y por 55 personas del estrato E (muy bajo). Según la edad, se entrevistaron 9 personas entre 20 y 24 años, 24 personas entre 25 y 29 años, 44 entre 30 y 34 años, 40 entre 35 y 39 años, 37 entre 40 y 44 años, 26 entre 45 y 49 años, 25 entre 50 y 54 años, 21 entre 55 y 59 años, 15 entre 60 y 64 años, 13 entre 65 y 69 años y 6 personas entre 70 y 74 años, correspondiendo esta última cifra a la máxima edad obtenida entre las personas encuestadas. La encuesta fue aplicada durante noviembre de 2004.

El instrumento utilizado correspondió a un cuestionario de respuesta cerrada con nueve preguntas. Las primeras tres estuvieron referidas a identificar el orden de preferencia de carne bovina, de ave y de cerdo, cada una con cuatro alternativas de respuesta. A continuación se consultó sobre la persona que decide y realiza la compra de carne bovina, cada una con cuatro posibles respuestas. La sexta pregunta consideró la frecuencia semanal de consumo con seis alternativas posibles. En forma posterior se preguntó sobre la forma preferida de presentación de la carne entre cinco alternativas. Finalmente, se presentaron cinco posibles cantidades de carne a adquirir por evento de compra y se consultó sobre el conocimiento que se posee sobre la preparación de la carne bovina, con cuatro posibles respuestas.

Los resultados fueron analizados mediante análisis de frecuencias, tablas de contingencia y el estadístico Chi cuadrado, que permite inferir si dos o más magnitudes de frecuencias de casos de la población pueden ser consideradas similares (Levin y Rubin, 1996).

En las tres primeras preguntas, los resultados de la segmentación por género (variable influyente en escala nominal dicotómica y variable de respuesta en escala ordinal) se analizaron mediante análisis estadístico para pruebas no paramétricas de dos muestras independientes utilizando la prueba $U$ de Mann Whitney. En el caso de las segmentaciones por estrato socioeconómico y grupo etario (variable influyente en escala nominal policotómica y variable de respuesta en escala ordinal) se realizó el análisis para pruebas no paramétricas con $\mathrm{K}$ muestras independientes correspondiente a la prueba de $\mathrm{H}$ de Kruskal Wallis. El uso de la prueba U de Mann
Whitney recomendada cuando sólo están involucradas dos muestras, y de la prueba $\mathrm{H}$ de Kruskal Wallis utilizada cuando se trata de más de dos muestras, permite determinar si muestras independientes han sido extraídas de la misma población o de distintas poblaciones con la misma distribución (Levin y Rubin, 1996). El paquete estadístico utilizado para la evaluación de la información obtenida correspondió al SPSS 11.0 (SPSS. Inc., USA) para Windows.

\section{RESULTADOS Y DISCUSIÓN \\ PREFERENCIAS DE CONSUMO DE DISTINTOS TIPOS DE CARNE}

La mayor proporción de encuestados prefirió carne bovina como primera opción de consumo habitual, seguida por la carne de pollo y de cerdo, las que fueron seleccionadas en mayor porcentaje como segunda y tercera opción (Cuadro 1). Esto concuerda con el estudio realizado por Narbona (1995) en la ciudad de Valdivia, donde 67\% de las personas indicó consumir más frecuentemente carne de vacuno y $32 \%$ señaló la carne de ave en primera opción. Sin embargo, ambos estudios se contraponen a las cifras entregadas por ODEPA (2005) que señalan que desde 1997 el consumo nacional de carne de ave ha superado a la carne bovina y a la tendencia decreciente en países europeos y EE.UU. detectada por Piggott et al. (1996), Rickertsen (1996) y USDA/ERS (2002). De la muestra, 2,3\% no prefirió ninguna de estas carnes como primera opción, señalando en su lugar carne de pavo y pescado.

No se detectaron diferencias estadísticas ( $\mathrm{p}>0,05)$ por segmento socioeconómico ni estrato de edad de los consumidores. Sólo la preferencia por carne bovina y de pollo relacionada con el género del encuestado presentó diferencias estadísticamente significativas $(\mathrm{p}<0,05)$; los hombres eligieron en una proporción más elevada la carne bovina como primera opción, y las mujeres en mayor porcentaje eligieron la carne de pollo. Estos resultados se contraponen a los obtenidos por Fundación Chile (2000) en Santiago, donde no se obtuvieron diferencias atribuibles al género del consumidor. Sin embargo, coinciden con reportes de la literatura internacional en relación a un menor consumo de carne bovina en consumidores de género femenino (MINTEL, 1997; MAFF, 1998; Harvey et al., 2001). 
Cuadro 1

Prioridad en la elección de carne bovina, de ave y de cerdo según género, estrato socioeconómico y grupo etario (\%). Temuco, noviembre de 2004

\begin{tabular}{|c|c|c|c|c|c|c|c|c|c|c|c|}
\hline \multirow{2}{*}{ Muestra } & \multicolumn{3}{|c|}{ Carne bovina } & \multicolumn{4}{|c|}{ Carne de pollo } & \multicolumn{4}{|c|}{ Carne de cerdo } \\
\hline & 1 & 2 & 3 & 1 & 2 & 3 & 4 & 1 & 2 & 3 & 4 \\
\hline Total & 62,3 & 30,0 & 7,7 & 31,8 & 59,0 & 8,4 & 0,8 & 7,9 & 13,7 & 74,1 & 4,3 \\
\hline Femenino & 57,4 & 33,5 & 9,1 & 35,6 & 57,7 & 6,1 & 0,6 & 8,3 & 10,4 & 77,1 & 4,2 \\
\hline Masculino & 73,5 & 21,7 & 4,8 & 23,7 & 61,8 & 13,2 & 1,8 & 7,0 & 20,9 & 67,4 & 4,7 \\
\hline $\mathbf{P}=$ & \multicolumn{3}{|c|}{0,011} & \multicolumn{4}{|c|}{0,022} & \multicolumn{4}{|c|}{0,367} \\
\hline $\mathrm{ABC} 1$ & 69,6 & 25,0 & 5,4 & 27,8 & 68,5 & 3,7 & 0,0 & 3,2 & 9,7 & 83,9 & 3,2 \\
\hline $\mathrm{C} 2$ & 69,5 & 25,4 & 5,7 & 27,1 & 62,7 & 8,5 & 1,7 & 5,9 & 11,8 & 79,4 & 2,9 \\
\hline $\mathrm{C} 3$ & 63,0 & 32,0 & 4,3 & 34,9 & 58,1 & 7,0 & 0,0 & 3,1 & 9,4 & 81,3 & 6,3 \\
\hline $\mathrm{D}$ & 50,0 & 32,5 & 17,5 & 34,4 & 46,9 & 18,8 & 0,0 & 21,1 & 21,1 & 57,8 & 0,0 \\
\hline $\mathrm{E}$ & 56,4 & 34,5 & 9,1 & 37,5 & 52,1 & 8,3 & 2,1 & 14,3 & 23,8 & 52,4 & 9,5 \\
\hline $\mathbf{P}=$ & \multicolumn{3}{|c|}{0,140} & \multicolumn{4}{|c|}{0,765} & \multicolumn{4}{|c|}{0,140} \\
\hline $1(20-24)$ & 44,4 & 55,6 & 0,0 & 50,0 & 37,5 & 12,5 & 0,0 & 16,7 & 16,7 & 66,7 & 0,0 \\
\hline $2(25-29)$ & 54,2 & 41,7 & 4,2 & 28,6 & 57,1 & 14,3 & 0,0 & 6,7 & 86,7 & 6,7 & 0,0 \\
\hline $3(30-34)$ & 51,2 & 4,19 & 7,0 & 41,9 & 48,8 & 7,0 & 2,3 & 8,3 & 8,3 & 75,0 & 8,3 \\
\hline $4(35-39)$ & 70,5 & 17,5 & 12,5 & 28,6 & 65,7 & 5,7 & 0,0 & 9,5 & 14,3 & 76,2 & 0,0 \\
\hline $5(40-44)$ & 78,4 & 18,9 & 2,7 & 18,2 & 72,7 & 6,1 & 3,0 & 4,8 & 9,5 & 76,2 & 9,5 \\
\hline $6(45-49)$ & 69,2 & 26,9 & 3,8 & 30,8 & 53,8 & 15,4 & 0,0 & 0,0 & 31,3 & 68,7 & 0,0 \\
\hline $7(50-54)$ & 56,0 & 32,0 & 12,0 & 37,5 & 50,0 & 12,5 & 0,0 & 10,0 & 30,0 & 60,0 & 0,0 \\
\hline $8(55-59)$ & 66,7 & 28,6 & 4,8 & 23,5 & 70,6 & 5,9 & 0,0 & 7,7 & 7,7 & 84,6 & 0,0 \\
\hline $9(60-64)$ & 66,7 & 20,0 & 13,0 & 21,4 & 71,4 & 7,1 & 0,0 & 33,3 & 16,7 & 50,0 & 0,0 \\
\hline $10(65-69)$ & 53,8 & 23,1 & 23,1 & 41,7 & 58,3 & 0,0 & 0,0 & 25,0 & 50,0 & 25,0 & 0,0 \\
\hline $11(70)$ & 50 & 50,0 & 0,0 & 50,0 & 50,0 & 0,0 & 0,0 & 0,0 & 0,0 & 100,0 & 0,0 \\
\hline $\mathbf{P}=$ & \multicolumn{3}{|c|}{0,393} & \multicolumn{4}{|c|}{0,675} & \multicolumn{4}{|c|}{0,393} \\
\hline
\end{tabular}

\section{LA COMPRA DE CARNE BOVINA}

En la muestra total (Cuadro 2) tanto la persona que compra la carne bovina como la que decide el corte a comprar correspondió mayoritariamente a la esposa, lo que concuerda con los resultados obtenidos por Harvey et al. (2001) en un estudio realizado con 1.065 consumidores en Inglaterra y por Verbeke y Vackier (2004) en una encuesta realizada a 625 consumidores en Bélgica. En segundo lugar se encuentra el jefe de hogar en ambos casos, con mayor importancia como comprador que como agente decisor. Sin embargo, la importancia de la mujer como compradora de carne resulta menor a lo detectado por Narbona (1995) en Valdivia y por Fundación Chile (2000) en Santiago, donde 72\% de los compradores de carne correspondió al género femenino (Fundación Chile, 2000).
Destaca la baja proporción de respuestas que señalaron decisión y compra compartida por parte de ambos cónyuges, como asimismo la baja participación de otros integrantes del grupo familiar. Esto se contrapone a lo determinado por Heiman et al. (2001) en Israel, donde las preferencias del grupo familiar influencian las decisiones de consumo de carne.

Existieron diferencias estadísticamente significativas $(\mathrm{p}<0,05)$ respecto a la persona que compra y decide la carne a comprar según el género del entrevistado. Tanto las mujeres como los hombres se atribuyeron en mayor medida el acto y decisión de compra, lo que resultó más marcado en el caso de la compra en los entrevistados varones y en la decisión de la carne a comprar en las mujeres. Respecto al estrato socioeconómico, sólo se detectaron diferencias estadísticas en la persona que decide la compra $(\mathrm{p}<0,05)$, mientras en el segmento $\mathrm{ABC} 1$ resulta muy elevada la partici- 
Cuadro 2

Persona que compra y decide la compra de carne bovina en supermercados de la ciudad de Temuco según género, estrato socioeconómico y grupo etario (\%). Temuco, noviembre de 2004.

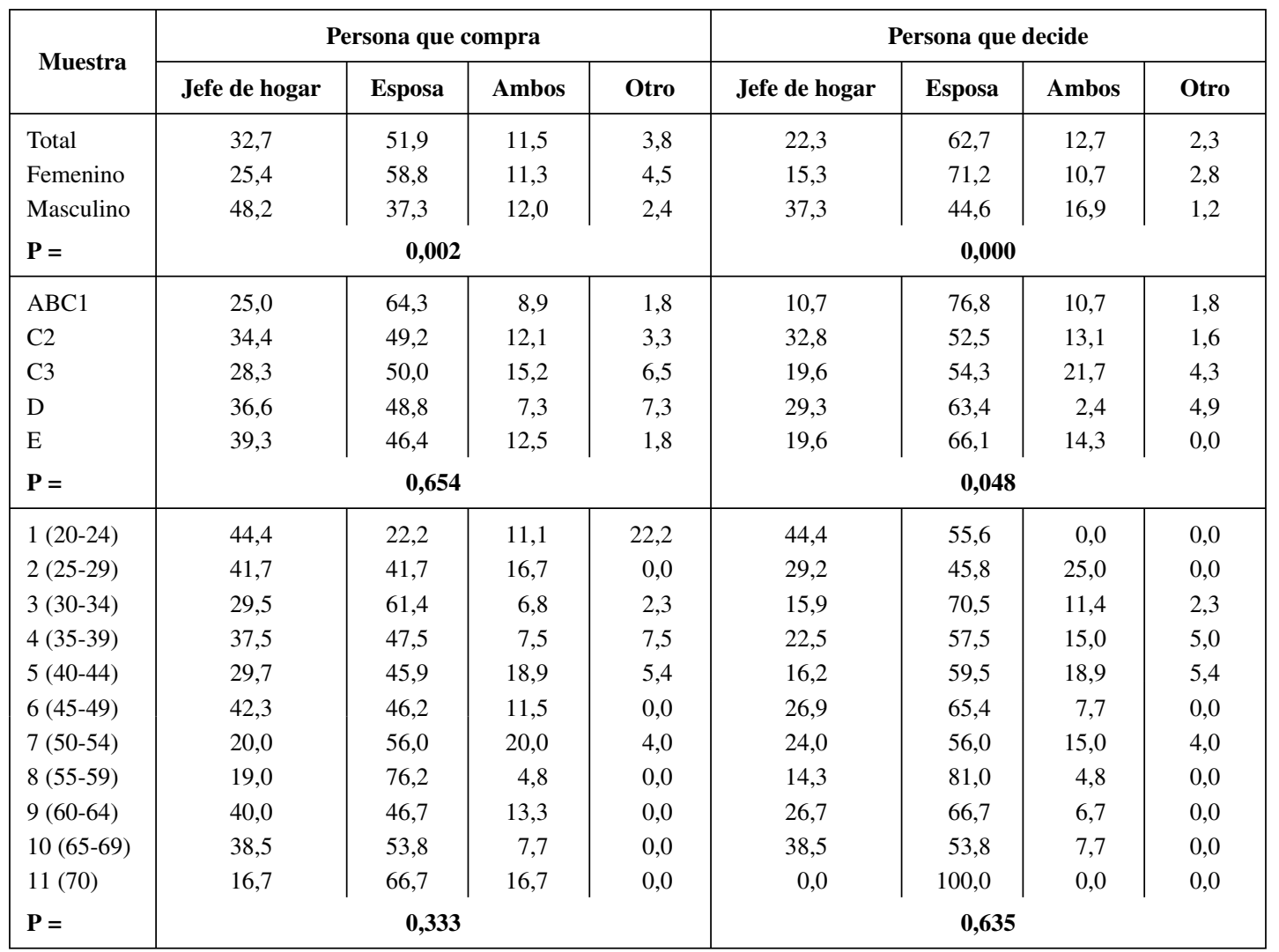

pación de la esposa, en C2 y D aumenta la presencia del jefe de hogar y en C3 destaca la mayor proporción de decisiones compartidas por ambos cónyuges. No existieron diferencias estadísticas $(\mathrm{p}>0,05)$ según la edad del entrevistado.

\section{FRECUENCIA DE CONSUMO DE CARNE BOVINA}

En la muestra total predominó el consumo de carne bovina tres y dos veces por semana (Cuadro 3 ), por lo que es posible afirmar que $65,4 \%$ de los encuestados consume este tipo de carne al menos dos veces a la semana. Este resultado concuerda con lo obtenido en consumidores de Valdivia por Narbona (1995). Las situaciones extremas como consumo diario y sólo los fines de semana o en ocasiones especiales bordearon cada una el $12 \%$.
Se obtuvieron diferencias significativas en la frecuencia semanal de consumo por estrato socioeconómico $(\mathrm{p}<0,05)$, dadas por la mayor proporción de encuestados del segmento $\mathrm{ABC} 1$ que consume carne bovina tres veces por semana y en forma diaria. Asimismo, y en forma congruente con el precio de la carne y el nivel de ingreso de los estratos, destacó la mayor proporción de entrevistados del segmento E que manifestó consumir este producto sólo una vez por semana o sólo los fines de semana, como también la mayor proporción de encuestados del estrato de menores ingresos que consume carne sólo en ocasiones especiales. Esto concuerda con los resultados obtenidos por Fundación Chile (2000) en Santiago, respecto a que la frecuencia de consumo de carne bovina depende del gasto realizado según el estrato socioeconómico. 
Cuadro 3

Frecuencia semanal de consumo de carne bovina según género, estrato socioeconómico y grupo etario (\%). Temuco, noviembre de 2004

\begin{tabular}{|c|c|c|c|c|c|c|}
\hline Muestra & Todos los días & $\begin{array}{l}\text { Tres veces/ } \\
\text { semana }\end{array}$ & $\begin{array}{c}\text { Dos veces/ } \\
\text { semana }\end{array}$ & $\begin{array}{l}\text { Una vez/ } \\
\text { semana }\end{array}$ & $\begin{array}{l}\text { Solo fines } \\
\text { de semana }\end{array}$ & $\begin{array}{c}\text { Solo ocasiones } \\
\text { especiales }\end{array}$ \\
\hline $\begin{array}{l}\text { Total } \\
\text { Femenino } \\
\text { Masculino } \\
\mathbf{P = \mathbf { 0 , 1 1 0 }}\end{array}$ & $\begin{array}{r}12,7 \\
14,1 \\
9,6\end{array}$ & $\begin{array}{l}39,6 \\
41,2 \\
36,1\end{array}$ & $\begin{array}{l}25,8 \\
24,3 \\
28,9\end{array}$ & $\begin{array}{r}10,4 \\
10,7 \\
9,6\end{array}$ & $\begin{array}{l}7,3 \\
6,2 \\
9,6\end{array}$ & $\begin{array}{l}4,2 \\
3,4 \\
6,0\end{array}$ \\
\hline $\begin{array}{l}\mathrm{ABC} 1 \\
\mathrm{C} 2 \\
\mathrm{C} 3 \\
\mathrm{D} \\
\mathrm{E} \\
\mathbf{P}=\mathbf{0 , 0 0 1}\end{array}$ & $\begin{array}{r}17,9 \\
11,5 \\
13,0 \\
9,8 \\
10,7\end{array}$ & $\begin{array}{l}57,1 \\
41,0 \\
34,8 \\
31,7 \\
26,8\end{array}$ & $\begin{array}{l}16,1 \\
32,0 \\
28,3 \\
17,1 \\
30,4\end{array}$ & $\begin{array}{r}7,1 \\
6,6,0 \\
13 \\
17,1 \\
10,7\end{array}$ & $\begin{array}{r}0,0 \\
1,6 \\
8,7 \\
19,5 \\
10,7\end{array}$ & $\begin{array}{l}1,8 \\
4,9 \\
2,2 \\
2,4 \\
8,9\end{array}$ \\
\hline $\begin{array}{l}1(20-24) \\
2(25-29) \\
3(30-34) \\
4(35-39) \\
5(40-44) \\
6(45-49) \\
7(50-54) \\
8(55-59) \\
9(60-64) \\
10(65-69) \\
11(70) \\
\mathbf{P}=\mathbf{0 , 3 6 9}\end{array}$ & $\begin{array}{r}0,0 \\
16,7 \\
18,2 \\
15,0 \\
5,4 \\
15,4 \\
8,0 \\
19,0 \\
6,7 \\
7,7 \\
16,7\end{array}$ & $\begin{array}{r}44,4 \\
37,5 \\
27,3 \\
40,0 \\
56,8 \\
53,8 \\
40,0 \\
47,6 \\
20,0 \\
30,8 \\
0,0\end{array}$ & $\begin{array}{r}44,4 \\
29,2 \\
22,7 \\
22,5 \\
27,0 \\
23,1 \\
20,0 \\
9,5 \\
53,3 \\
23,1 \\
50,0\end{array}$ & $\begin{array}{r}0,0 \\
8,3 \\
18,2 \\
7,5 \\
8,1 \\
0,0 \\
20,0 \\
4,8 \\
13,3 \\
7,7 \\
33,3\end{array}$ & $\begin{array}{r}0,0 \\
4,2 \\
4,5 \\
7,5 \\
2,7 \\
7,7 \\
12,0 \\
14,3 \\
6,7 \\
23,1 \\
0,0\end{array}$ & $\begin{array}{r}11,1 \\
4,2 \\
9,1 \\
7,5 \\
0,0 \\
0,0 \\
0,0 \\
4,8 \\
0,0 \\
7,7 \\
0,0\end{array}$ \\
\hline
\end{tabular}

No se detectaron diferencias significativas según el género y la edad del consumidor ( $\mathrm{p}>0,05)$, lo que se contrapone a los resultados de West et al. (2001) en relación a que la frecuencia de compra de carne bovina es influida por la edad y sexo de los consumidores canadienses. Paralelamente, este resultado difiere de lo reportado por MINTEL (1997), MAFF (1998) y Becker et al. (1998) respecto a que el consumo de carne bovina tiende a disminuir con la edad.

\section{FORMA EN LA CUAL LOS CONSUMIDORES PREFIEREN COMPRAR LA CARNE}

La forma de preferencia está dada principalmente por el envase que presenta la carne y la forma en que se entrega. La forma correspondió a carne fresca en corte o en vara y congelada, los envases al vacío y en bandeja. En la muestra total (Cuadro 4) predominaron claramente las preferencias por la carne fresca en cortes sin envasar, resultado que se contrapone a lo obtenido por Fundación Chile (2000) en Santiago respecto a la amplia aceptación que tienen las carnes en bandeja en los compradores de supermercados de esta ciudad.

Se obtuvieron diferencias estadísticamente significativas según el género y el estrato socioeconómico del consumidor $(\mathrm{p}<0,05)$. A pesar de que tanto hombres como mujeres prefieren en mayor proporción la carne por corte, las mujeres se inclinaron en mayor medida por la carne en bandeja y los hombres mostraron mayor preferencia por carne envasada al vacío y congelada. Los estratos socioeconómicos altos y medios presentaron mayor preferencia por carne en corte y los segmentos bajos por carne en bandeja y congelada, lo que se relaciona con el menor precio que presenta este último tipo de carne. La mayor preferencia de los estratos altos y medios por carne 
Cuadro 4

Forma en la cual los consumidores prefieren comprar la carne en supermercados según género, estrato socioeconómico y grupo etario (\%). Temuco, noviembre de 2004.

\begin{tabular}{|c|c|c|c|c|c|}
\hline \multirow{2}{*}{ Muestra } & \multicolumn{5}{|c|}{ Forma de compra de la carne } \\
\hline & Vara & Vacío & Corte & Bandeja & Congelada \\
\hline Total & 5,4 & 6,5 & 70,8 & 11,9 & 5,4 \\
\hline Femenino & 5,6 & 4,0 & 73,4 & 14,1 & 2,8 \\
\hline $\begin{array}{l}\text { Masculino } \\
\mathbf{P}=\mathbf{0 , 0 0 4}\end{array}$ & 4,8 & 12,0 & 65,1 & 7,2 & 10,8 \\
\hline $\mathrm{ABC} 1$ & 10,7 & 5,4 & 75,0 & 8,9 & 0,0 \\
\hline $\mathrm{C} 2$ & 4,9 & 4,9 & 78,7 & 6,6 & 3,3 \\
\hline $\mathrm{C} 3$ & 8,7 & 4,3 & 73,9 & 13,0 & 0,0 \\
\hline $\mathrm{D}$ & 0,0 & 12,2 & 61,0 & 19,5 & 4,9 \\
\hline $\begin{array}{l}E \\
\mathbf{P}=\mathbf{0 , 0 0 1}\end{array}$ & 1,8 & 7,1 & 58,9 & 14,3 & 17,9 \\
\hline $1(20-24)$ & 11,1 & 0,0 & 44,4 & 22,2 & 22,2 \\
\hline $2(25-29)$ & 12,5 & 12,5 & 62,5 & 4,2 & 8,3 \\
\hline $3(30-34)$ & 0,0 & 4,5 & 77,3 & 13,6 & 4,5 \\
\hline $4(35-39)$ & 5,0 & 15,0 & 62,5 & 12,5 & 5,0 \\
\hline $5(40-44)$ & 0,0 & 5,4 & 81,1 & 8,1 & 5,4 \\
\hline $6(45-49)$ & 0,0 & 5,4 & 81,1 & 8,1 & 5,4 \\
\hline $7(50-54)$ & 11,5 & 3,8 & 73,1 & 7,7 & 3,8 \\
\hline $8(55-59)$ & 0,0 & 8,0 & 68,0 & 16,0 & 8,0 \\
\hline $9(60-64)$ & 4,8 & 0,0 & 90,5 & 4,8 & 0,0 \\
\hline $10(65-69)$ & 6,7 & 0,0 & 73,3 & 20,0 & 0,0 \\
\hline $\begin{array}{l}11(70) \\
\mathbf{P}=\mathbf{0 , 0 5 6}\end{array}$ & 21,3 & 7,7 & 61,5 & 7,7 & 0,0 \\
\hline
\end{tabular}

en vara, se podría asociar al mayor equipamiento de congeladores que posee este tipo de hogares, donde conservar la carne para consumos posteriores. No se obtuvieron diferencias estadísticas según el estrato etario del entrevistado ( $\mathrm{p}>0,05)$.

\section{CANTIDAD DE CARNE ADQUIRIDA POR EVENTO DE COMPRA}

La cantidad comprada es otra variable que determina la necesidad de consumo. En la muestra total (Cuadro 5) es posible distinguir dos grupos de compra, el primero formado por $68,7 \%$ de los encuestados que adquiere $2 \mathrm{~kg}$ o menos por evento de compra destacando los consumidores que compran entre 1 y $2 \mathrm{~kg}$. El segundo grupo, formado por $30,8 \%$, compra $3 \mathrm{~kg}$ o más destacando los consumidores que adquieren entre 3 y $5 \mathrm{~kg}$. Estos resultados indican que la cantidad comprada por evento de compra en la ciudad de Temuco es mayor que la observada en Santiago por Fundación Chile (2000), pero similar a la obtenida por Vacarezza (1996) en Argentina.

Se detectaron diferencias estadísticamente significativas $(\mathrm{p}<0,05)$ por estrato socioeconómico, destacando la mayor proporción de consumidores del estrato ABC 1 que compró entre 3 y $5 \mathrm{~kg}$ y de consumidores de $\mathrm{C} 2$ que adquirieron sobre $5 \mathrm{~kg}$. Paralelamente, se observaron mayores proporciones de consumidores de los estratos D y E que compraron entre $1 / 2$ y $1 \mathrm{~kg}$ por evento de compra, mientras que la mayor proporción de encuestados que adquiere menos de $1 / 2 \mathrm{~kg}$ correspondió al estrato de menores ingresos.

No se detectaron diferencias significativas según el género y la edad del consumidor ( $\mathrm{p}>0,05)$. Esto último nuevamente se contrapone a los resultados 


\section{Cuadro 5}

Cantidad de carne bovina adquirida por evento de compra en supermercados y grado de conocimiento del consumidor con respecto a los usos de diferentes cortes al momento de cocinarlos, según género, estrato socioeconómico y grupo etario (\%). Temuco, noviembre de 2004.

\begin{tabular}{|c|c|c|c|c|c|c|c|c|c|}
\hline \multirow{2}{*}{ Muestra } & \multicolumn{5}{|c|}{ Cantidad comprada (kg) } & \multicolumn{4}{|c|}{ Conocimiento uso de los cortes } \\
\hline & $<1 / 2$ & $1 / 2-1$ & $1-2$ & $3-5$ & $>5$ & Todos & La mayoría & Solo algunos & Ninguno \\
\hline Total & 3,8 & 13,8 & 51,5 & 20,8 & 10,0 & 16,9 & 32,3 & 40,4 & 10,4 \\
\hline Femenino & 5,1 & 13,6 & 52,0 & 19,8 & 9,6 & 15,8 & 34,5 & 43,5 & 6,2 \\
\hline Masculino & 1,2 & 14,5 & 50,6 & 22,9 & 10,8 & 19,3 & 27,7 & 33,7 & 19,3 \\
\hline $\mathbf{P}=$ & \multicolumn{5}{|c|}{0,385} & \multicolumn{4}{|c|}{0,008} \\
\hline $\mathrm{ABC} 1$ & 1,8 & 8,9 & 48,2 & 28,6 & 12,5 & 21,4 & 55,4 & 21,4 & 1,8 \\
\hline $\mathrm{C} 2$ & 1,6 & 13,1 & 52,5 & 16,4 & 16,4 & 9,8 & 36,1 & 47,5 & 4,9 \\
\hline $\mathrm{C} 3$ & 6,5 & 13,0 & 52,2 & 21,7 & 6,5 & 26,1 & 26,1 & 41,3 & 6,5 \\
\hline D & 2,4 & 17,1 & 65,9 & 12,2 & 0,0 & 19,5 & 19,5 & 51,2 & 7,3 \\
\hline E & 8,9 & 17,9 & 39,3 & 21,4 & 10,7 & 10,7 & 17,9 & 42,9 & 26,8 \\
\hline $\mathbf{P}=$ & \multicolumn{5}{|c|}{0,037} & \multicolumn{4}{|c|}{$\mathbf{0 , 0 0 0}$} \\
\hline $1(20-24)$ & 11,1 & 22,2 & 44,4 & 11,1 & 11,1 & 11,1 & 22,2 & 44,4 & 22,2 \\
\hline $2(25-29)$ & 4,2 & 25,0 & 50,0 & 8,3 & 12,5 & 8,3 & 41,7 & 37,5 & 12,5 \\
\hline $3(30-34)$ & 4,5 & 15,9 & 52,3 & 20,5 & 6,8 & 22,7 & 29,5 & 34,1 & 13,6 \\
\hline $4(35-39)$ & 5,0 & 15,0 & 47,5 & 22,5 & 10,0 & 0,0 & 30,0 & 60,0 & 10,0 \\
\hline $5(40-44)$ & 0,0 & 13,5 & 48,6 & 16,2 & 21,6 & 21,6 & 37,8 & 32,4 & 8,1 \\
\hline $6(45-49)$ & 7,7 & 3,8 & 53,8 & 26,9 & 7,7 & 15,4 & 34,6 & 42,3 & 7,7 \\
\hline $7(50-54)$ & 0,0 & 16,0 & 56,0 & 24,0 & 4,0 & 16,0 & 36,0 & 44,0 & 4,0 \\
\hline $8(55-59)$ & 4,8 & 4,8 & 61,9 & 23,8 & 4,8 & 14,3 & 33,3 & 42,9 & 9,5 \\
\hline $9(60-64)$ & 0,0 & 13,3 & 66,7 & 20,0 & 0,0 & 46,7 & 6,7 & 33,3 & 13,3 \\
\hline $10(65-69)$ & 7,7 & 15,4 & 38,5 & 23,1 & 15,4 & 30,8 & 30,8 & 30,8 & 7,7 \\
\hline $11(70)$ & 0,0 & 0,0 & 33,3 & 50,0 & 16,7 & 16,7 & 50,0 & 16,7 & 16,7 \\
\hline $\mathbf{P}=$ & \multicolumn{5}{|c|}{0,528} & \multicolumn{4}{|c|}{0,281} \\
\hline
\end{tabular}

obtenidos por MINTEL (1997), MAFF (1998) y Becker et al. (1998) en relación a una disminución del consumo de carne bovina con la edad.

\section{CONOCIMIENTO DEL CONSUMIDOR DE LOS USOS DE LOS CORTES AL MOMENTO DE COCINARLOS}

Aproximadamente 50\% de los encuestados de la muestra total manifestó poseer un alto conocimiento de los usos de la carne, al considerar en conjunto aquellos que conocen todos y la mayoría de los usos. La fracción de encuestados que no conoce ningún corte ni sus usos resulta menor a lo determinado por Narbona (1995) en Valdivia, donde $41 \%$ no conocía el uso que se le puede dar a los distintos cortes de carne bovina. No obstante, resulta destacable en el presente estudio que en conjunto $50,8 \%$ de los encuestados sólo conoce algunos o ningún uso de un producto que, en la mayoría de los casos, se consume al menos dos veces por semana.

Sólo se detectaron diferencias significativas según el género y estrato socioeconómico del consumidor $(\mathrm{p}<0,05)$. Si bien la proporción de hombres que declaró conocer el uso de todos los cortes de la carne bovina fue superior, también lo fue la fracción que no conoce ninguno. Sin embargo, considerando los consumidores de ambos géneros que conocen todos y la mayoría de los usos, se obtienen proporciones similares $(50,3 \%$ mujeres y $47 \%$ hombres), habiendo sido esperable un mayor grado de conocimiento en las mujeres quienes en mayor medida resultaron ser las personas que deciden y compran la carne bovina. Las diferencias 
por segmento socioeconómico correspondieron principalmente a la mayor proporción de consumidores de $\mathrm{ABC} 1$ que declaró conocer la mayoría de los usos de la carne, la superior fracción del estrato D que sólo conoce algunos usos y el elevado porcentaje de consumidores del segmento de más

\section{LITERATURA CITADA}

BECKER, T., BENNER, E. AND GLITSCH, K. 1998. Summary report on consumer behaviour towards meat in Germany, Ireland, Italy, Spain, Sweden and the United Kingdom: results of a consumer survey. Working paper FAIR-CT950046, Department of Agricultural Markets and Agricultural Policy, The University of Hohenheim, Göttingen.

BURTON, M., TOMLINSON, M. AND YOUNG, T. 1994. The impact of BSE on the demand for beef and other meats in Great Britain. Applied Economics, 18: 687-693.

CHALFANT, J. A. AND ALSTON, J. M. 1988. Accounting for changes in tastes. Journal of Politics and Economics, 96: 391-410.

FUNDACIÓN CHILE. 2000. Cadena Agroalimentaria de la Carne Bovina en Chile. Disponible en http.//www.fundacionchile. cl/pls/portal/docs/page/portal_corporativo/administracion_del_ contenido/agroindustria/publicaciones/cadenas\%20bovino. pdf. Conectado el 5 de agosto de 2002.

GILBERT, F. W. AND WARREN, W. E. 1995. Psychographic constructs and demographic segments. Psychology and Marketing, 12: 223-237.

GREATOREX, M.AND MITCHELL, V. W. 1994. Modeling consumer risk reduction preferences from perceived loss. Journal of Economic Psychology, 15: 669-685.

HARVEY, J., ERDOS, G. AND CHALINOR, S. 2001. The relationship between attitudes, demographic factors and perceived consumption of meats and other proteins in relation to the BSE crisis: a regional study in the United Kingdom. Health, Risk \& Society, 3: 181-197.

HEIMAN, A., JUST, D., MCWILLIAMS, B., AND ZILBERMAN, D. 2001. Incorporating family interactions and socioeconomics variables into family production functions: the case of demand of meats. Agribusiness, 17: 455-468.

INE. 1998. INSTITUTO NACIONAL DE ESTADÍSTICAS. (ON LINE). VI CENSO AGROPECUARIO, 1997. Disponible en: http://www.ine.cl/ine/canales/chile_estadistico/estadisticas_economicas/agropecuarias/xls/2005/censoagropecuario. xls. Conectado el 30 de abril de 2000.

INE. 2001. Quinta encuesta de presupuestos familiares (agosto 1996 - julio 1997). Disponible en: http://www.ine.cl/ine/ canales/chile_estadistico/estadistica_laborales/vencuesta/ volumen1/xls/cuunvoun.xls. Conectado el 17 de diciembre de 2001.

LEVIN, R. Y D. RUBIN. 1996. Estadística para Administradores. Prentice Hall, Hispanoamericana S.A. Sexta edición. Juárez. México. 1018 p. bajos ingresos que no conoce ningún uso. Estos resultados concuerdan tanto con la frecuencia de compra como con las cantidades adquiridas por evento de compra obtenidas en el presente estudio, ambos factores asociados al ingreso percibido por los estratos socioeconómicos.

MAFF. 1998. National Food Survey 1998. Crown Copyright.

MINTEL, 1997. Red meats, Mintel Food and Drink, July 1997.

MAINO, M., K. KÖBRICH Y J. SEPÚLVEDA. 2000. Caracterización de la demanda de carne bovina en el sector de restaurantes. En V Congreso de Economía Agraria "La Agricultura en una Economía Internacionalizada", Santiago, Chile. p. 270-280.

MINTEL, 1997. Red meats, Mintel Food and Drink, July 1997.

NARBONA, C. 1995. Estudio sobre la conducta del consumidor y sus cambios como consecuencia de la aplicación de la tipificación de carne bovina; discriminación por calidad (Ingeniero en Alimentos). Universidad Austral de Chile. Valdivia, Chile. 150 p.

ODEPA. 2005. Disponible en http://www.odepa.cl. Conectado el 15 de diciembre de 2005

PIGGOTT, N., CHALFANT, J., ALSTON, J. AND GRIFFITH, G. 1996. Demand response to advertising in the Australian meat industry. American Journal of Agricultural Economics, 71: 253-261.

RESURRECCION, A. V. A. 2003. Sensory aspects of consumer choices for meat and meats products. Meat Science, 66:11-20.

RICKERTSEN, K. 1996. Structural change and the demand for meat and fish in Norway. European Review of Agricultural Economics, 23: 316-330.

SCHEAFFER, R., MENDWENHALL, W. Y OTT, L. 1996. Elementos de Muestreo. Grupo editorial Iberoamericana S.A. Primera edición. Ciudad de México, México. 345 p.

USDA/ECONOMIC RESEARCH SERVICE. 2002. Changing consumer demands create opportunities for U.S. food system. Food Review, 25(1): 19-22.

VACAREZZA, M. 1996. Proceso de decisión de compra del consumidor de carne vacuna del barrio céntrico de la ciudad de Tendil en Argentina. 5 de octubre 2002. 34 p. Disponible en http://read.adm.ufrgs.br/read10/artigo/artigo2.PDF. Conectado el 20 de agosto de 2002.

VERBEKE, W. AND VACKIER, I. 2004. Profile and effects of consumer involvement in fresh meat. Meat Science 67: 159-168.

WEST, G., LARUE, B., TOUIL, C. AND SCOTT, S. 2001. The perceived importance of veal meat attributes in consumer choice decisions. Agribusiness, 17(3) 365-382. 